\title{
Tsafon
}

Revue d'études juives du Nord

$82 \mid 2021$

Enjeux esthétiques dans la littérature après Auschwitz

\section{Enjeux esthétiques dans la littérature après Auschwitz}

Andrée Lerousseau

\section{(2) OpenEdition}

1 Journals

Édition électronique

URL : https://journals.openedition.org/tsafon/4139

DOI : $10.4000 /$ tsafon.4139

ISSN : 2609-6420

Éditeur

Association Jean-Marie Delmaire

Édition imprimée

Date de publication : 1 décembre 2021

Pagination : 7-14

ISSN : 1149-6630

\section{Référence électronique}

Andrée Lerousseau, «Enjeux esthétiques dans la littérature après Auschwitz », Tsafon [En ligne], 82 |

2021, mis en ligne le 01 décembre 2021, consulté le 12 février 2022. URL : http://

journals.openedition.org/tsafon/4139; DOI : https://doi.org/10.4000/tsafon.4139

Tsafon. Revues d'études juives du Nord 


\section{Dossier}

rassemblé et présenté par Andrée Lerousseau

Enjeux esthétiques dans la littérature après Auschwitz 
Tsafon 82 
«Les événements d'Auschwitz, du ghetto de Varsovie, de Buchenwald ne supporteraient certainement pas une description d'ordre littéraire ; la littérature n'y était pas préparée et ne s'est pas donné les moyens d'en rendre compte ${ }^{1}$, confiait Brecht. C'est ce que confirme des années plus tard André Schwarz-Bart, sur le ton de l'affirmation liée à sa propre expérience : «Le thème d'Auschwitz ne se prête pas à la littérature (création littéraire en général, roman ou théâtre) $»^{2}$. Élie Wiesel, quant à lui, voyait dans le simple fait de poser la question de "l'Holocauste comme source d'inspiration littéraire " l'expression d'une aporie, accompagnant son propos relatif aux limites de la représentation littéraire d'un impératif éthique à connotation religieuse et effectuant par là-même une sacralisation de l'événement: «Un roman sur Maïdanek frôle le blasphème. Est un blasphème $»^{3}$. La littérature perdrait-elle ainsi toute légitimité face à l'événement ?

\footnotetext{
${ }^{1}$ Cité dans Dayan Rosenman Anny, "Entendre la voix du témoin », Mots, $\mathrm{n}^{\circ}$ 56, septembre 1998. La Shoah : silence... et voix, sous la direction de Anne Croll et Annette Wieviorka, DOI : https://doi.org/10.3406/mots. 1998.2362.

www.persee.fr/doc/mots_0243-6450_1998_num_56_1_2362-, p. 1, note 2 (dernière consultation 01/ 11/2021).

${ }^{2}$ Voir infra la dernière partie de l'article de Francine Kaufmann pour les citations de cet auteur.

${ }^{3}$ Élie Wiesel, (1990), « The Holocaust as literary inspiration », dans Dimensions of the Holocaust, Illinois, Northwestern University Press, 1983, p. 7 : «"The Holocaust as Literary Inspiration" is a contradiction in terms [...]. A novel about Majdanek is about blasphemy. Is blasphemy ».
} 
La porte d'Auschwitz a beau demeurer infranchissable ${ }^{4}$, la littérature s'obstine. Il y a bien, pour paraphraser Maurice Blanchot, une « écriture du désastre », " née au cœur même de la guerre, [et qui] ne cesse d'être en mouvement depuis son origine », ainsi que le souligne Juliette Adams (voir infra), mutations qui passent, ainsi que l'illustre ce dossier, par la levée progressive d'un certain nombre de tabous. « Ce qui aurait pu être une fin s'est avéré un point de départ », constate Annette Wieviorka dans sa préface aux Alphabets de la Shoah, ouvrage majeur d'Anny Dayan Rosenman $^{5}$. La question dès lors ne porte pas tant sur la possibilité d'une littérature « de » et «sur» la Shoah, que sur la représentation littéraire adéquate d'une réalité longtemps qualifiée d'indicible. C'est peut-être en ce sens qu'il faut comprendre l'allégation d'Adorno, sur laquelle le philosophe lui-même devait revenir : «Écrire un poème (après Auschwitz) est barbare, et ce fait affecte même la connaissance qui explique pourquoi il est devenu impossible d'écrire aujourd'hui un poème $»^{6}$. Si les œuvres de Nelly Sachs ou de Paul Celan, pour ne citer qu'eux, ont apporté un démenti immédiat à cette affirmation un peu abrupte, résonnant de prime abord comme un interdit, elles n'en démontrent pas moins l'impossibilité dans laquelle se trouve l'écrivain - et tout aussi bien l'artiste - de s'adonner à la création et de (se) représenter le monde comme si rien ne s'était passé : «la réalité d'Auschwitz ne se prête à aucun arrangement esthétique traditionnel», écrit Schwarz-Bart, et tout «arrangement» renouant avec l'esthétique traditionnelle peut légitimement, au regard de l'éthique, être qualifié de « barbare ». Mais comment dès lors appréhender cette réalité ?

Dans son article qui retrace l'itinéraire d'André Schwarz-Bart, Francine Kaufmann met en lumière l'unité d'une œuvre profondément humaniste et universaliste dénonçant sous toutes ses formes «l'univers concentrationnaire ». Prix Goncourt en 1959, Le dernier des Justes, véritable « ovni dans le ciel du Nouveau Roman alors en vogue », répond à un double impératif : prononcer le kaddish à la mémoire de ceux qui furent privés de sépulture et restaurer un monde rayé de la carte dans sa

\footnotetext{
${ }^{4}$ Voir infra ces lignes d'André Schwarz-Bart: «La vérité, c'est qu'il n'a jamais su franchir la porte d'Auschwitz pour y accompagner les siens. Il a frappé mille fois à cette porte, mais elle ne s'est jamais ouverte. Il a cru la franchir avec Haïm, mais c'était une illusion ».

${ }^{5}$ Anny Dayan Rosenman, Les alphabets de la Shoah, Paris, CNRS Éditions, 2007.

${ }^{6}$ Theodor Adorno, Prismes. Critique de la culture et de la société, trad. par Geneviève et Rainer Rochlitz, Paris, Éditions Payot et Rivages, 2010, p. 30-31.
} 
triple dimension historique, culturelle et spirituelle. Le cycle antillais dont l'écrivain entame plus tard la rédaction, s'il est une façon d'aborder la Shoah " de biais », au même titre que les fragments inédits où l'auteur adopte une stratégie du dédoublement, répond à un même souci de restitution, voire de réparation. Renouant à quelques années de distance avec la « saga identitaire » et avec la représentation d'un « héros-peuple » derrière lequel l'auteur s'efface, Schwarz-Bart, précurseur en ce sens de la " mémoire multidirectionnelle», y rend hommage à un autre groupe exposé aux humiliations et s'attache une fois de plus à célébrer la vie, tant il est vrai que ce n'est qu'à partir de la vie bafouée ou éradiquée que l'on prend pleinement la mesure des crimes perpétrés. Quant aux notes et fragments inédits, condensé des doutes, des questionnements, des angoisses qui taraudent l'écrivain ou l'artiste confronté à la Shoah, mais qui n'en posent pas moins des limites infranchissables ou à ne pas franchir sous peine de sombrer dans le voyeurisme ou l'abjection, c'est peu dire qu'ils suffiraient à eux seuls à introduire ce dossier, et nous remercions infiniment Francine Kaufmann pour le partage de ces écrits et tenons à exprimer toute notre gratitude à Simone Schwarz-Bart, l'épouse de l'écrivain, pour son autorisation à les publier.

Le Dernier des justes « a ouvert aux lettres françaises des espaces de sensibilité pour elles jusque-là inconnus », confiait Piotr Rawicz, auquel Juliette Adams consacre son étude, et avec la publication de son roman Le Sang du ciel en 1961 se poursuit la mue entamée avec SchwarzBart, une mutation consistant à s'affranchir des témoignages bruts et des récits génocidaires confinant parfois au martyrologue pour témoigner autrement. Ancien déporté, Rawicz opte délibérément pour la littérature, la poétisation et la fictionnalisation de la Shoah. Pas à pas, dans une brillante analyse des stratégies narratives et discursives mises en œuvre, Juliette Adams suit le langage qui se cherche, explore avec sensibilité les espaces ouverts par Rawicz dans sa quête d'une "nouvelle poétique » et d'une esthétique fondée sur «un pacte de réciprocité entre l'art et la souffrance » qui se double d'un questionnement sur l'identité, sur la part troublante et ambivalente de l'être humain, et sur le statut du survivant. Comment trouver ce « tiers espace » entre l'avant et l'après, qui permet de vivre?

Cette interrogation est au cœur de la réflexion d'Atinati Mamatsashvili portant sur La lune noircie (2009) d'Anise Koltz et Les miroirs d'Ostende (1978) de Paul Willems, récit poétique et pièce de théâtre transportant le lecteur dans "l'ère de 'l'après' ", dans cette 
« temporalité frauduleuse d'une histoire qui se continue », selon les termes empruntés à Alexis Nouss. La question ne porte d'ailleurs pas tant - et là réside toute l'originalité de l'approche - sur la possibilité de renouer avec « l'avant », de restaurer une continuité, que sur celle de faire « coïncider » « l'après » avec l'idée que l'on s'en faisait avant. À moins de s'adonner à une mise en scène fondée sur la supercherie, ce jeu de miroirs s'avère impossible. Le corps du survivant s'interpose, revenant indésirable témoignant de la condition de l'homme après Auschwitz, de sa « superfluité », et d'un monde irrémédiablement marqué par la dislocation et la désagrégation (des corps, des lieux et du langage), un monde qu'Atinati Mamatsashvili place d'emblée sous le signe du théâtre de Beckett, dans un rejet de l'esthétique traditionnelle.

Si, dans l'après-Auschwitz, tout « arrangement » avec les normes esthétiques ayant cours auparavant ne peut être que frauduleux, il serait indécent d'aborder la réalité même d'Auschwitz par ce biais : « Shakespeare n'est pas possible à Auschwitz », note Schwarz-Bart, avant d'ajouter : " La survie comme but unique pourrait seulement convenir à un théâtre de bêtes plus ou moins sourdes, aveugles et muettes : taupes, insectes, herbivores et carnivores ». Nous voici ainsi transportés dans l'univers des Cannibales (1968) de George Tabori, qui oppose un humour noir, déjà présent chez Rawicz, à la «fossilisation» et à la « mythologisation $»^{7} \mathrm{du}$ discours sur l'holocauste, dans un recours au burlesque provoquant un rire «amer», un rire pour conjurer l'effroi, comme une " bouée de sauvetage », ainsi que le souligne Andrea Grassi. Tabori rejoignait ainsi ces autres briseurs de tabou que furent à la fin des années soixante Romain Gary ou Edgar Hilsenrath. En dépit de sa fascination pour le théâtre de Tabori, Juan Mayorga choisit, presque quatre décennies plus tard, un tout autre angle d'approche, beaucoup moins subversif (faut-il y voir un reflet de l'époque ?), dans Himmelweg (2003). D'où l'intérêt de la mise en regard des deux pièces dont le dénominateur commun serait, ainsi que l'avance l'auteur, de congédier le « rire satisfait ». « Représentation d'une représentation », en contact permanent avec l'archive (ainsi que cela est démontré à partir d'un choix de citations particulièrement pertinent), Himmelweg, dont Andrea Grassi propose une lecture à la lumière de Kafka et d'Hannah Arendt, renoue avec les codes du théâtre de l'absurde et interroge par ce biais les rapports de forces invisibles qui lient les victimes et les bourreaux.

\footnotetext{
${ }^{7}$ Voir infra les remarques de Mayorga à propos du théâtre de Tabori.
} 
La question de l'identité et de l'altérité, si elle n'est pas ici saisie en termes d'ambivalence troublante, est au centre de l'étude de Jiaojiao Xia. Partant d'un constat de Freud relatif aux traces mnésiques ancrées dans le corps, l'auteure apporte un éclairage nouveau, par la convergence de sources puisées dans l'histoire des idées, sur Histoire d'une vie (1999) et Le garçon qui voulait dormir (2010) d'Aharon Appelfeld, appréhendant ces écrits autofictionnels à partir de la philosophie de l'identité personnelle de Ricœur, de la philosophie de la mémoire de Bergson, et de la notion de « mémoire affective » développée par Paul Sollier. Précise et rigoureuse, l'analyse est convaincante et permet de cerner au plus près l'avènement de la "mémoire corporelle » qui est au principe même de la création chez Appelfeld. "Les souvenirs prennent littéralement corps» au creux du texte, le geste - ou tout aussi bien le refus momentané de tout mouvement corporel - portant témoignage au présent et en direction d'un futur, au cœur même de l'écriture, du traumatisme vécu.

Les contributions à ce dossier, se proposant de mettre en évidence un certain nombre d'enjeux esthétiques dans la littérature après Auschwitz, illustrent à notre sens une double exigence.

La première concerne la nécessité qui se fit ressentir, à un point donné de la temporalité instaurée par Auschwitz, de ce que nous serions tentée de nommer un engagement de la littérature, appelée à se faire le relai de la mémoire d'un événement qui ne cesse de nous interroger sur ce que nous sommes, à s'emparer de cet « objet », non pas pour se substituer aux témoignages directs, mais pour dire autrement, par le biais d'une approche plus « oblique », cet « indicible » que la parole, achoppant sur le silence ou se heurtant à la porte close, n'a de cesse de tenter de cerner, et entrant ainsi en résistance contre l'oubli.

La seconde, qui fait figure d'un impératif, consiste en l'obligation faite à la littérature de la Shoah de se renouveler au fil du temps " pour continuer d'exister », ainsi que le relève Juliette Adams, dans une prise en compte en particulier des exigences des lecteurs. Au fur et à mesure que le temps passe, la question, soulevée par Schwarz-Bart dans ses notes, de la réception de cette littérature par les jeunes générations et les générations à venir se fait de plus en plus pressante. Comment en effet, par quels (nouveaux) détours, enseigner à nos enfants ce qui, aux yeux de SchwarzBart, constitue l'héritage d'Auschwitz, à savoir « le plus grand désespoir et le plus parfait amour [de la vie] »? Cet héritage, qui peut sembler hautement paradoxal, s'inscrit au plus profond de la tradition juive : faire 
malgré tout le choix de la vie, dans le maintien d'une mémoire vivante et qui prend corps, par la convocation des visages des disparus, en vertu même peut-être de cette "sur-vie» qui ne parvient pas à congédier complètement le terme. C'est le plus grand défi lancé aux artisans de la mort. 